Proc. International Conference on Networking - ICN 2005, Reunion Island, LNCS, April, 2005, Springer-Verlag, Vol. 3420, pp. 438-448

\title{
ESCORT: Energy-efficient Sensor Network Communal Routing Topology Using Signal Quality Metrics
}

\author{
Joel W. Branch, Gilbert G. Chen, and Boleslaw K. Szymanski \\ Rensselaer Polytechnic Institute \\ Department of Computer Science \\ Troy, New York, U.S.A. \\ \{brancj, cheng3, szymansk\}@cs.rpi.edu
}

\begin{abstract}
ESCORT aims at decreasing the energy cost of communication in dense sensor networks. We employ radio frequency (RF) signal quality assessment in forming communities of redundant nodes. These communities avoid spanning regions of environmental interference to preserve the routing fidelity of the network. ESCORT is routing protocol-independent and conserves energy by alternating redundant nodes' radio duty cycles. Simulation demonstrates that ESCORT enables nodes to deactivate their radios more than $60 \%$ of the time while sustaining acceptable communication performance.
\end{abstract}

\section{Introduction}

Advances in hardware and communications technologies, coupled with the increased need for on-demand mobile computing, are fueling the advances in pervasive computing. An essential component of this new computing paradigm is wireless sensor networks (WSNs), which collect and analyze information describing environmental phenomena. Some interesting examples of WSN applications are described in [7], [8].

Unfortunately, WSNs come with inherent challenges. One is that tiny sensor nodes are resource-constrained devices, providing limited storage, processor, and battery capacity. Another one is costly transceiver operation that strains nodes' batteries. Finally, transient wireless links threaten an application's integrity. Therefore, WSN algorithms should promote energy-efficiency while sustaining application quality. The above challenges serve as motivation for our work. Further motivation arises from an observation that each WSN is tuned to a very specific problem, and thus, no individual WSN protocol will be applicable in all scenarios; this includes routing protocols. Therefore, methods for enhancing energy-efficiency of multiple routing protocols must be adopted.

A well-accepted method of energy conservation in WSNs, and one which we follow, is the selective deactivation of nodes' radios. Generally, radio operation uses huge amounts of energy, as represented by the transmit/receive/sense/idle ratio for a Crossbow MICA2DOT sensor mote [4]: $75 \mathrm{~mW} / 24 \mathrm{~mW} / 15 \mathrm{~mW} / 81 \mu \mathrm{W}$ (assuming a $3 \mathrm{~V}$ power source). As this ratio demonstrates, radio operation is generally the most costly activity of sensor nodes. 
This paper presents ESCORT, which represents our research on the novel use of RF signal quality assessment (SQA) to cluster wireless sensor nodes based on connectivity and spatial separation. This allows a community of redundant nodes to function as a single virtual routing entity and thus, operate transparently under most routing protocols. Establishing sleep schedules within the communities then saves energy. Our contribution regards the use of SQA, which helps ESCORT mitigate the effect of packet loss and control the extent of community formation, preserving the connectivity of the overall network.

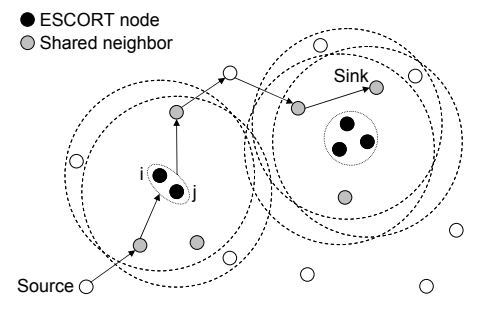

Fig. 1. ESCORT topology applied to a small wireless sensor network

Fig. 1 shows ESCORT's effect on a WSN in which communities of redundant nodes are formed (indicated by the dotted circles), and shared neighbors, residing within the communitys' intersected transmission regions (indicated by dashed circles), are established to ensure that communities maintain only bi-directional links with neighboring nodes. The example path is routed through the community on the left, in which either node $i$ or $j$ may forward the traffic depending on their transceiver states.

Before continuing, we state some fundamental assumptions concerning ESCORT. First, sensor board components operate independently from transceivers and remain powered over all states. Second, all inter-node links are bi-directional. Third, nodes exhibit no mobility. Additional assumptions are stated as needed.

The remainder of this paper starts with a description of the ESCORT approach in Section 2 and a detailed description of the actual algorithm in Section 3. Section 4 presents a performance evaluation. Section 5 provides a discussion of related work and Section 6 concludes the paper.

\section{The ESCORT Approach}

\subsection{RF Signal Quality Assessment}

We define RF signal quality as a combination of two separate metrics: link quality and signal strength. We describe their uses below. Both factors help to determine the selection of redundant sensor nodes while sustaining acceptable application-level performance. 
Link Quality. We use link quality assessment to form communities of nodes with equivalent routing functionality for two main reasons. One, healthy links promote energy-efficient packet delivery. Lal et al. support this assertion by demonstrating that extensive retransmissions over faulty links waste transmission energy [6]. Two, healthy intra-community links provide robust intra-group coordination that preserves the layer of transparency under the selected routing algorithm, ensure proper determination of sleep schedules and help to reliably share routing state information.

Fig. 2 shows the result of considering intra-community link quality in constructing node communities. The obstructions cause poor link quality between two groups of nodes. As a result, separate communities have been formed on either side of the obstructions. Without link quality assessment, one community might have formed, exhibiting poor coordination due to loss of ESCORT control packets. Later, we describe where link quality assessment fits into the ESCORT algorithm.

Designing link quality assessment algorithms is beyond the scope of this paper. We assume use of a technique proposed in [6], in which link quality is graded via packet delivery rate and signal-to-noise ratio measurements. The authors observe that in energy-constrained networks, where nodes save energy via radio deactivation, the quality of the wireless links are not known a priori to packet transmission. Thus, a low-cost initialization phase is used during which nodes periodically wake up to measure link quality.

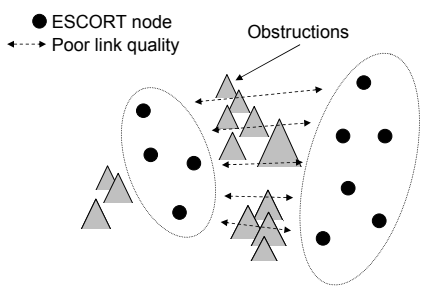

Fig. 2. Effect of link quality assessment in community formation

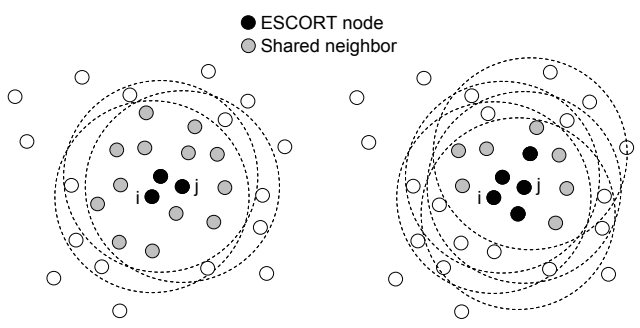

Fig. 3. Comparative effect of using different signal strength thresholds on community formation

Signal Strength. Another important metric for communal topology formation is the spatial separation between nodes. It can be measured in various ways, perhaps the most intuitive being the use of GPS coordinates. However, even though GPS may be used by the application layer, because of its energy requirements and cost, we decided not to require its use. Instead, we use the received signal strength (RSS) for distance estimation and thus the second metric of signal quality.

Other distance measurement methods are available (e.g., time difference of arrival and angle of arrival), but we adopt RSS for its relatively low implementation overhead. We do not convert the actual signal strength into distance, since we need a comparative measure and not absolute distance itself.

Signal strength enables us to control the tradeoff between energy savings and the network connectivity. Adding nodes to community decreases its reach-ability. Fig. 3 illustrates the difference between using smaller and larger RSS thresholds to form 
communities. In the left community, nodes $i$ and $j$ cooperate in soliciting nearby nodes to join their community. We assume that all candidates exhibit acceptable link quality with both nodes $i$ and $j$. With large RSS thresholds (such that nodes registering RSS measurements above a pre-defined value may join the community) only the closest neighbors gain membership. However, on the right, nodes $i$ and $j$ relax the RSS threshold, allowing the community to grow larger. As a result, the number of shared neighbors eligible for synchronous communication decreases. Since communal nodes' sleep time increases with community size, the tradeoff between potential energy savings and network connectivity is obvious from the figure.

One might assume that signal strength may also be used to predict packet loss behavior, thus eliminating the need link quality assessment. However, in [11], experiments disprove the perceived strong correlation between signal strength and packet loss, finding that not all links with high RSS exhibit low packet loss. Thus, we do not rely on the use of RSS for measuring link quality.

\section{The ESCORT Algorithm}

\subsection{Initialization}

RF Signal Quality Assessment. Initialization begins with each node assessing the quality of its wireless links. As previously stated, we expect to use a method such as that described in [6] to assess link quality. We propose parallelizing RSS assessment with link quality assessment since it would be beneficial to gauge links' signal strengths over multiple samples to obtain average values for each link, $\overline{R S S}$. We also assume that this sub-phase will account for variations in the environment. Neighbors exhibiting intolerable signal quality are excluded as neighbors all together.

Topology Establishment. In this sub-phase, each node identifies an initial partner. Since multiple neighbors will probably exhibit healthy link quality, the neighbor displaying the highest $\overline{R S S}$ value is selected as the initial partner. We also do this in the interest of maintaining network connectivity in the unlikely case that the initial community can not further expand. A JOIN_REQUEST packet is sent to the potential partner and pairing is established when two nodes select each other as partners.

The next step involves community expansion. For each initial node pair, $i$ and $j$, the node with the highest ID is designated as the coordinator (assume this to be $i$ ) and thus takes responsibility for coordinating the community's expansion. Included with each JOIN_REQUEST is the respective node's inner-neighbor set, $A$. Given $i$ 's full neighbor set, $B_{i}$, and the global link quality threshold, $L Q_{-}$THRESH, $A_{i}$ is defined as:

$$
A_{i}=\left\{x: x \in B_{i} \text { and } L Q_{i x}>L Q_{-} T H R E S H\right\}
$$

where $L Q_{i x}$ is the link quality rating between $i$ and $x$. Also included in $A_{i}$ is each neighbor's $\overline{R S S}$ value. Given a predefined global threshold, RSS_THRESH, $i$ selects 
neighbors from both sets, $A_{i}$ and $A_{j}$, to form a potential community set, $P C$, defined as:

$$
P C=\left\{x: x \in A_{i}, A_{j} \text { and } \overline{R S S_{i x}}, \overline{R S S_{j x}}>R S S_{-} T H R E S H\right\}
$$

where $\overline{R S S_{i x}}$ and $\overline{R S S_{j x}}$ are the $\overline{R S S}$ values of node $x$ registered at $i$ and $j$ respectively. $P C$ represents the set of candidates used for community expansion.

Continuing, the coordinator node solicits each node in the set $P C$ to join its community by transmitting JOIN_REQUEST2 packets. In the event that multiple JOIN_REQUEST2 packets are received, the $\overline{R S S}$ values of the requesting nodes are used as tie-breakers with the highest $\overline{R S S}$ value winning. This helps optimize the inter-node distance within the formed communities. Solicited nodes then send JOIN_REPLY2 packets to their chosen coordinator and all nodes belonging to a particular community adopt a pseudo ID matching that of the coordinator node (the original ID is not discarded). This formation of one semantic node allows transparent interaction between the routing and ESCORT protocol layers since all nodes in a community are now receptive to a shared identity.

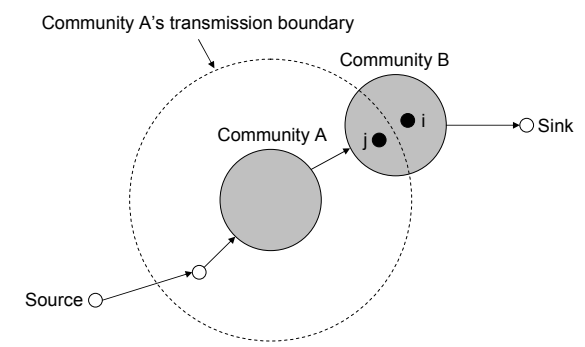

Fig. 4. An ill-conditioned ESCORT community causing an inconsistent routing state

Routing protocol faults may occur if ESCORT produces inconsistent states. This is illustrated by the ill-conditioned community $B$ in Fig. 4, two of whose members $i$ and $j$ lie on either side of community $A$ 's communication boundary. Suppose during the routing protocol's path discovery phase, $j$ 's radio is active, while the rest of community $B$ 's radios are deactivated. Subsequently, $A$, perceived as one node to the network layer, arbitrarily selects $B$ as its "next hop" on some constructed path, due to $j$ 's active state. However, at a later time, $i$, which lies outside of $A$ 's communication range, will become active and the path segment $A-B$ will cause significant packet loss.

We prevent this scenario by utilizing the community members' individual neighbor sets constructed in the initialization phase. Proceeding community establishment, the intersection of all of the members' neighbor sets is calculated by each member. Afterwards, each node knows the resultant reach-ability of the entire community, and precautions are taken to prohibit communication with excluded neighboring nodes or communities via transmission of IGNORE packets. At this point, communities can effectively communicate as singular entities without concerns of asynchrony. 


\subsection{Runtime}

ESCORT's runtime behavior is driven by leader election and state-sharing. The leader node is the one that handles routing for its community during a given duty cycle. Routing protocol state-sharing is conducted between duty cycles to ensure that new leaders route packets appropriately using updated information. To avoid packet loss, ESCORT control messages should use a channel separate from those used by the routing layer for communication. Such separation makes the updated route information delivery rate independent of the application traffic loads that may introduce channel contention. If state-sharing were disrupted due to this condition, the application packet delivery rate would decrease because of possibly incomplete routing information.

Leader Election. Leader election is designed to be efficient, fair, and fault-tolerant, promoting graceful network degradation. To balance the community's workload, the node with the most residual energy at the end of a duty cycle is chosen as the active node for the next cycle. The original coordinator node is the only node known to share high quality links with the rest of its community. Thus, it conducts leader election and state-sharing. This duty, coupled with normal routing duties would place an unfair burden on the coordinator nodes, so they are currently exempt from routing duties.

After the duty cycle time, $T_{d c}$, expires, all nodes enter the election state and send a VOTE packet, containing the node's residual energy, to the coordinator. The last active node also includes its energy dissipation rate over the last duty cycle. The coordinator then selects the node with the highest residual energy as the new leader and broadcasts its ID to the community.

Fairness is further achieved by the calculation of $T_{d c}$. So that all nodes dissipate energy at approximately the same rate, an exponential average is used to predict the dissipation rate in the next duty cycle based on past rates. Thus, if a node $j$ wins the election, its predicted dissipation rate, $D R_{j}$, is calculated by the coordinator as:

$$
D R_{j}=\alpha\left(D R_{i}\right)+(1-\alpha)(\tau)
$$

where $D R_{i}$ is the dissipation rate of the last active node during the last cycle, $\tau$ stores the average over the history of operation, and $\alpha$ controls the responsiveness to recent history. $T_{d c}$ is then calculated by:

$$
T_{d c}=\frac{p \times I E_{j}}{D R_{j}}
$$

where $I E_{j}$ is the initial energy of $j$ and $p$ is the percentage of $I E_{j}$ to be expended in the next duty cycle. $T_{d c}$ is then broadcast to the entire community.

State Sharing. In the state-sharing phase, the last active node sends the new leader its routing layer state information (e.g., forwarding tables, counters, etc.) to maintain routing fidelity. We note that the last active node continues to forward packets on 
behalf of the community until the end of this phase. Any changes to the state between the time state information is transmitted and received at the new leader should be negligible to performance. If necessary, additional interaction between the routing and ESCORT layers may handle significant route updates, but we leave this for future research. After the routing state is transferred, the new leader remains active while all other nodes sleep for the calculated duty cycle time.

\section{Performance Evaluation}

In this section, we present an analysis of ESCORT's performance using the SENSE simulator [16]. Our main intent was to compare various performance metrics of a wireless multi-hop sensor network with and without ESCORT applied. The following performance metrics, which we examine, have evolved as standard ratings in the literature for benchmarking WSNs:

Packet delivery rate: Percent of total end-to-end DATA packets successfully delivered

Packet delay: End-to-end time incurred for DATA packet delivery

Sleep rate: Percent of total time a node spends sleeping

Energy consumption: Energy consumed by a node throughout the simulation

Network lifetime: Time needed for $70 \%$ of the path nodes ${ }^{1}$ to drain their batteries

\subsection{Simulation Framework and Environment}

We used four components to model the WSN protocol stack: application, network, $M A C$, and physical. The application component implemented a bursty traffic model. The network component defined the routing protocol. The MAC component provided an implementation of the IEEE 802.11 wireless protocol standard. The physical component simulated the radio. SENSE also has a channel component, which simulates propagation effects in the wireless communication medium, and a battery component, that models energy consumption. Finally, we designed the ESCORT component, placing it between the network and MAC components.

Our energy consumption model is derived from the power specifications for the Crossbow MICA2DOT MPR510CA sensor mote [4]. For most experiments, nodes start with an initial energy of $1 * 10^{4} \mathrm{~J}$. For the experiments in which we measure network lifetime, we change the initial energy to $1 * 10^{3} \mathrm{~J}$ in order to decrease lengthy simulation times.

All experiments were executed on a virtual test-bed of size $800 \mathrm{~m} * 400 \mathrm{~m}$, on which nodes were randomly placed. The population ranged from 30 to 80 nodes; we tested in increments of 10 nodes. We altered the SENSE channel component to model obstructions. At this point, obstructions are represented by rectangular entities with a thickness in the range of 5-50m. Obstructions were placed randomly near the center

\footnotetext{
${ }^{1}$ We only consider nodes lying on route paths because performance of those is the most effected by ESCORT.
} 
of the test-bed so as to maintain a variety of routes between the sources and sink, which are positioned on opposite sides of the test-bed. For this initial study, we assumed that any significant line-of-site obstructions rendered the signal unsuitable for communication, eliminating its use by ESCORT.

The free space propagation model was used throughout all simulations. All sets of simulations were executed twice: once with nodes using a transmission range of $250 \mathrm{~m}$ and once using a range of $300 \mathrm{~m}$. The size of the DATA packet's payload was 512 b. Routes were established using the AODV routing protocol [9]; ESCORT was then applied to AODV for performance testing. For simplicity, traffic (source and sink) nodes were not clustered by ESCORT.

For each simulation type, ten trials were executed for each population. Each simulation ran for 50,000 units of simulated time. In referring to Equations 3 and 4, we set the values of $\alpha$ and $p$ to 0.9 and 0.001 respectively

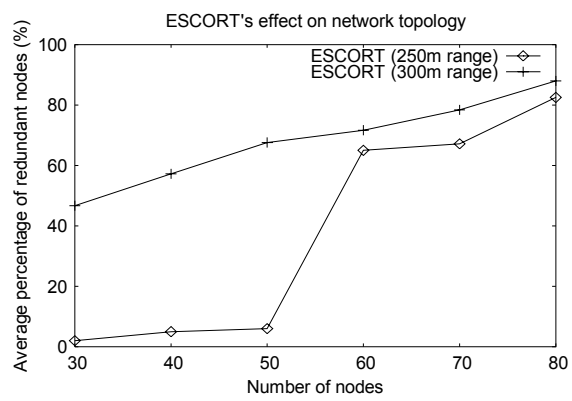

Fig. 5. Transmission range and node density impact on ESCORT's energy savings

\subsection{Topology Characteristics}

Fig. 5 illustrates how ESCORT's effect on network topology increases along with network density. Fig. 5 specifically shows that for a larger transmission range, ESCORT's effect on the network is more persistent as the network density increases, solidifying the potential for the network to save energy. For a smaller transmission range, ESCORT displays a similar advantage only after a particular threshold, lying somewhere between a population of 50 and 60 nodes. These results lend to the idea that ESCORT is especially beneficial for those networks with larger transmission ranges. We note that the RSS_THRESH values were adjusted so as to allow communities to grow larger along with the population size. This is because at smaller populations, larger communities risk having little or no neighbors to route to. Dynamically selecting the RSS_THRESH is a subject of future research. 


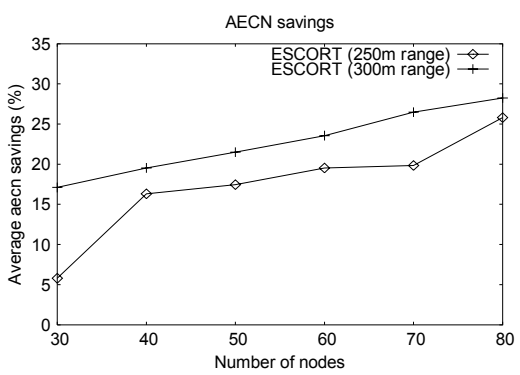

Fig. 6. Average AECN Savings

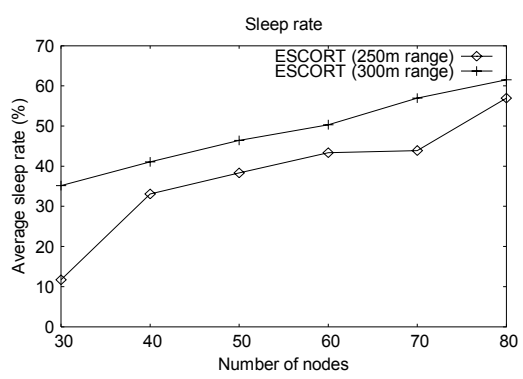

Fig. 7. Average sleep time percentage

\subsection{Energy Savings}

We assess energy savings by two measurements. First, we inspect the average energy consumption per node (aecn). aecn, adopted from [18], is defined as follows:

$$
\text { aecn }=\frac{E_{0}-E_{t}}{n \times t}
$$

where $E_{0}$ and $E_{t}$ is the initial energy and the residual energy at time $t$ (end of simulation), respectively, for $n$ total nodes. Fig. 6 illustrates the average aecn savings achievable with ESCORT, showing up to $25 \%$ reduction for the $250 \mathrm{~m}$ case and $28 \%$ reduction for the $300 \mathrm{~m}$ case. The second metric we use is sleep rate. Fig. 7 shows that ESCORT allows nodes to sleep up to more than $55 \%$ of the time for the $250 \mathrm{~m}$ case and more than $60 \%$ of the time for the $300 \mathrm{~m}$ case. These two metrics together show that while ESCORT allows a significant amount of sleep time, factors influencing energy savings depend also on the nodes' transmission range and other radio power specifications.

ESCORT also contributed a significant improvement to network lifetime. For the $250 \mathrm{~m}$ case, ESCORT achieved up to an approximate $38 \%$ (at 80 nodes) increase in network lifetime over the case using just AODV. For the $300 \mathrm{~m}$ case, similar increase was achieved: $36 \%$ with 80 nodes. These results highlight the significance of ESCORT's energy savings that are essential for prolonged WSN operation.

\subsection{Packet Delivery Performance}

ESCORT's energy-efficiency is achieved without impacting the packet delivery rate, which held at about $99 \%$ across all experiments. ESCORT's impact on another packet delivery metric, delay, shown in Fig. 8 and Fig. 9, is also rather insignificant. Additional delay is no more than approximately 1.3 seconds for the cases of $250 \mathrm{~m}$ and $300 \mathrm{~m}$ transmission ranges. This additional delay grows slowly with the increase of node population. Overall, these results show ESCORT's ability to sustain application performance even for large node densities. 
Many other attempts at energy savings showed that packet delivery performance usually decreases as a result of increased energy savings. Our results show that ESCORT can decrease the energy expense of communication with minimum tradeoffs in quality of service.

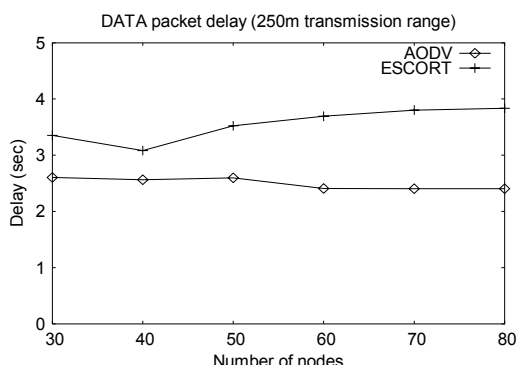

Fig. 8. Average DATA packet delivery delay for the $250 \mathrm{~m}$ case

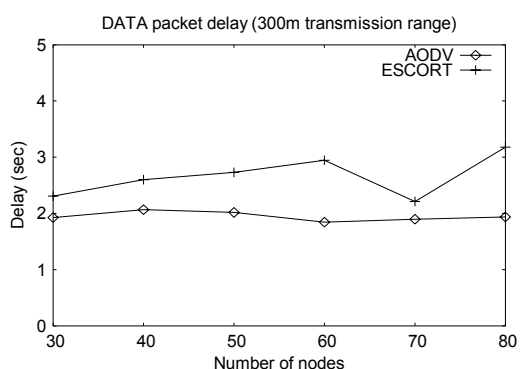

Fig. 9. Average DATA packet delivery delay for the $300 \mathrm{~m}$ case

\section{Related Work}

ESCORT is most closely related to topology-based frameworks. The LEACH [5] protocol uses a cluster-based topology in which the cluster-head role is periodically rotated to fairly distribute energy dissipation. Within the clusters, data fusion is used to reduce the traffic load to the base station. Similar to ESCORT, nodes use signal strength measurements to decide which cluster to join. However, the authors focus on direct transmission, rather than multi-hop, WSNs. In GAF [10], nodes divide the network into a grid using GPS coordinates. Grid squares are composed of equivalent nodes which are all able to directly communicate with neighbors in adjacent squares. This work is similar to ESCORT. However, GAF makes no provisions for signal quality in forming communities. ESCORT also forgoes the expense of using GPS technology. Span [2] and ASCENT [1] are similar in nature. Both protocols focus on keeping enough nodes awake to maintain established backbones in the network. In Span, a node wakes up and joins the network only if its adjacent neighbors can not directly communicate with each other. ASCENT makes a similar effort, but causes nodes to join the network only when the quality of the link between its neighbors falls below a predefined threshold. ESCORT is closer related to ASCENT because of its attention given to link quality metrics. However, ESCORT assumes a more pro-active role constructing its neighborhoods, avoiding tradeoffs in packet loss and latency.

\section{Conclusion}

As we have shown, ESCORT effectively provides energy-efficient routing for wireless sensor networks. Our simulation results give an indication of the significant 
amount of energy that can be saved with ESCORT. Furthermore, ESCORT is fully distributed and scalable. Hence, our research is important to increasing the feasibility for WSNs.

We have identified several directions for our future research. One is researching ways in which ESCORT may accommodate asynchronous links. Second is to research how to make ESCORT adapt to dynamic environmental conditions, such as sporadic node failures. This is very challenging, as no previous work has focused on maintaining well-formed communities (considering signal quality assessment) in dynamic environments. Another aspect of adaptation involves dynamic node arrivals. We plan to extend ESCORT to dynamically incorporate new nodes into the routing framework during runtime operation. The third direction is to design mechanisms for waking up sleeping nodes in the case that they are summoned for data retrieval. This might involve the use of a separate low-energy radio cycle. Finally, we plan to research techniques to rotate the functionality of the coordinator node during run-time operation. This presents another significant challenge as the coordinator node must share high-quality wireless links which all of its community members.

\section{References}

1. Cerpa, A. and Estrin, D.: ASCENT: adaptive self-configuring sensor networks topologies. Proc. IEEE INFOCOM '02 (2002) 1278-1287

2. Chen, B., Jamieson, K., Balakrishnan, H., and Morris, R.: Span: An energy-efficient coordination algorithm for topology maintenance in ad hoc wireless networks. Proc. ACM MobiCom '01 (2001) 85-96

3. Chen, G., Branch, J., Pflug, M., Zhu, L., and Szymanski, B.: SENSE: A wireless sensor network simulator. In: Szymanski, B., Yener, B. (eds.): Advances in Pervasive Computing and Networking. Springer, New York (2004) 249-267

4. Crossbow Technology, Inc.: MPR/MIB mote hardware users manual [Online]. Available: http://www.xbow.com

5. Heinzelman, W.R., Chandrakasan, A., and Balakrishnan, H.: Energy-efficient communication protocol for wireless microsensor networks. Proc. HICSS '00 (2000) 3005-3014

6. Lal, D., Manjeshwar, A., Herrmann, F., Uysal-Biyikoglu, E., and Keshavarzian, A.: Measurement and characterization of link quality metrics in energy constrained wireless sensor networks. Proc. IEEE GLOBECOM '03 (2003) 446-452

7. Lee, J. and Hashimoto, H.: Controlling mobile robots in distributed intelligent sensor network. IEEE Transactions in Industrial Electronics, Vol. 50, No. 5, (2000) 890-902

8. Mainwaring, A., Polastre, J., Szewczyk, R., Culler, D., and Anderson, J.: Wireless Sensor Networks for Habitat Monitoring. Proc. ACM WSNA'02 (2002) 88-97

9. Perkins, C., Belding-Royer, E., and Das, S.: RFC 3561-ad hoc on-demand distance vector (AODV) routing (2003) [Online]. Available: http://www.faqs.org/rfcs/rfc3561.html

10. Xu, Y., Heidemann, J., and Estrin, D. Geography-informed energy conservation for ad hoc routing. Proc. ACM MobiCom '01 (2001) 70-84

11. Zhao, J. and Govindan, R.: Understanding packet delivery performance in dense wireless sensor networks. Proc. SenSys '03 (2003) 1-13 